\title{
Il ruolo delle riviste letterarie italiane nella prima metà dell'Ottocento
}

\author{
Gisele Batista da Silva ${ }^{1}$ \\ Universidade Federal do Rio de Janeiro \\ gisabats@gmail.com
}

RIASSUNTO: In questo saggio analizziamo la creazione, lo sviluppo e il ruolo di alcune riviste letterarie italiane sorte nella prima metà del XIX secolo, tra le quali si distaccano Biblioteca Italiana, Il Conciliatore e Antologia. Nel nostro studio mettiamo in evidenza le relazioni politiche e culturali tra i loro fondatori e $\mathrm{i}$ vari interlocutori e consideriamo altresì la partecipazione e il contributo di queste riviste alla diffusione della questione romantica o alla critica della stessa - questione particolarmente dibattuta nella Italia di quegli anni. Esaminiamo inoltre l'influenza esercitata da queste riviste sui circoli di letterati italiani, svolgendo un ruolo chiave nella presa di posizione di questi intellettuali, in particolare per quanto riguarda le questioni culturali dell'Italia del XIX secolo.

Parole-chiave: Romanticismo italiano. Riviste letterarie. Biblioteca Italiana. Il Conciliatore. Antologia.

ABSTRACT: The purpose of this article is to analyse the creation, performance and role of some Italian literary magazines created in the first half of the 19th century, most lyre presented by the Biblioteca Italiana, Il Conciliatore and Antologia. It emphasizes the political-cultural relations established between its founders and interlocutors and shows the participation and the contribution of these periodicals in the diffusion of the romantic issue that gained prominence and importance in Italy in the analysed period. It have also examines some of the changes on Italian writer's circles influenced by these magazines, which revealed that they have played a fundamental role in shaping intellectual positions regarding the cultural issues of nineteenthcentury Italy.

Keywords: Italian Romanticism. Literary magazines. Biblioteca Italiana. Il Conciliatore. Antologia.

${ }^{1}$ Dottoressa in Letteratura italiana (UFRJ), ha il Master in Letteratura brasiliana (UERJ) e in Didattica dell'italiano a stranieri (Ca' Foscari). Attualmente è docente di Lingua italiana presso la UFRJ e partecipa al GT di Anpoll "Estudos da tradução". 
Tentando una riforma era nostro debito di indicare con precisionerigorosa le vere fonti e i soggetti della nuova letteratura [...] Noi volevamo congiungere in alleanza la letteratura d'Italia collo spirito del Secolo.

Alessandro Manzoni Notizia sul Romanticismo in Italia

In una lettera scritta a Charles-Claude Fauriel nel 1820 Alessandro Manzoni condivide con l'amico francese la sua valutazione degli effetti e delle risonanze del movimento romantico in Italia. Per il letterato milanese il romanticismo italiano non rappresentò l'introduzione di "un intero sistema di teorie Germaniche", ma fu costruito "pezzo per pezzo e senza verun precedente disegno" (VIGORELLI, 2008, p.201), e ciò inizialmente avvenne nelle pagine dei periodici Biblioteca Italiana e Il Conciliatore. La riflessione di Manzoni sugli obiettivi del movimento romantico in Italia mostra la peculiare situazione della penisola rispetto al resto dell'Europa, in quanto tale romanticismo conservava ancora in un "falso italianismo"(VIGORELLI, 2008 , p. 203) la giustificazione per nascondere uno stato di ignoranza e di inerzia, che generavano immobilità e arretratezza culturale. Tra gli equivoci elencati dallo scrittore e drammaturgo lombardo in quella lettera, evidenziamo l'idea secondo la quale il popolo italiano, più di ogni altro, era dotato di una notevole immaginazione, caratteristica che gli concedeva, in linea teorica, una posizione incomparabilmente superiore rispetto al resto dell'Europa.Questo tipo di pensiero, rappresentato da "frasi parassiti" diffuse nei circoli accademici (VIGORELLI, 2008, p. 204), rifletteva un contesto storico-sociale piuttosto complesso (e del quale era anche un riflesso) ${ }^{2}$, in cui veniva implicata una convivenza conflittuale tra tradizione e innovazione. In risposta a questo contesto, il progetto manzoniano desiderava esaminare le fonti, allontanandosi dalle dichiarazioni fuorvianti ${ }^{3}$, e rileggere la letteratura italiana a partire dallo

${ }^{2 “[. . .] ~ l a ~ L e t t e r a t u r a, ~ e s s e n d o ~ i n e r e n t e ~ a l l o ~ S t a t o ~ s o c i a l e ~ d i ~ u n ~ p o p o l o, ~ n e ~ s e g u e ~ n e c e s s a r i a m e n t e ~ l e ~}$ fasi, ne raccoglie lo spirito, e ne diviene la più bella espressione" (VIGORELLI, 2008, p. 204).

3Tra cui: "non solo i quattro grandi nostri Classici, ma tutta l'infinita serie sinonima de' trecentisti e de' cinquecentisti, toccarono l'apice della perfezione"; "quanto più una produzione nuova somiglia 
scenario europeo in trasformazione, in virtù del quale la tradizione era rivalutata e nuovi percorsi e obiettivi della letteratura nazionale venivano proposti.

Della consapevolezza di queste trasformazioni si ha traccia pure in scritti di altri autori; grazie anche ad essi la discussione in merito al rinnovamento dei contenuti e degli aspetti culturali prendeva forma in Italia: laLettera semiseria di Grisostomo al suo figliuolo di Berchet; il Sermone sulla mitologia, di Monti; la Lettre à M. Chanvetsurl'unité de temps et de lieudans la tragédie, di Manzoni, o ancora i vari testi del "filosofo del romanticismo italiano", Ermes Visconti ${ }^{4}$. Tali manifestazioni critiche rappresentarono la vocedi differenti aspirazioni letterarie e diffusero anche i progetti ideologici di quegli intellettuali, in quanto essi erano direttamente coinvolti nel compito di dare attributo, consistenza e visibilità a un gapesistente nella storia (letteraria) italiana: un'identità.

Tra queste manifestazioni, Di Breme desiderava intensamente l"Italia italiana e non Latina"5 in lettera scritta a Diodata Saluzzo nel maggio 1816, in opposizione al livellamento culturale tra greci, latini e italiani. Tale rivendicazionerappresentava una sorta di motto spartiacque nella querelle degli antichi e moderni, che valeva a distinguere tralaparte dell'Italia di orientamento moderno e un'altra di tendenza classicista, con tutte le implicazioni ideologiche che questapolemicapotrebbe suggerire. L'arena del dibattito, che si venne ad originare, ebbe sede in periodici e in varie riviste letterarie, tra le quali in particolare, laBiblioteca italiana, Il Conciliatore e Antologia, di cui tratteremo brevemente in questo studio.

Milano, capitale del Regno d'Italia tra il 1805 e il 1814, il cuorerispettabile della vita intellettuale italiana, assimilò durante questo periodo il meglio di ciò

un'antica [...] tanto è migliore”; “noi discendendo da' Romani siamo il Popolo primogenito, il popolo nobile dell'Europa" (VIGORELLI, 2008, p. 203).

${ }^{4}$ Il soprannome dato dal suo amico Goethe esprimeva l'ammirazione dello scrittore tedesco per il talento critico di Visconti, nella speranza di porre fine alla controversia romantica in Italia e ai conflitti che ne derivavano. Cf. FUBINI, 1953, p. 10.

${ }^{5}$ In questa lettera, Di Breme chiede autorizzazione di pubblicare la sua difesa alle considerazioni di Madame de Staëlsull'Italia. (FUBINI,1953, p.16). 
che venne prodotto in materia intellettuale e culturale. Si profilò come urgente, pertanto, creareun periodico che filtrasse e diffondesse queste produzioni e che si affermasse, inoltre, come luogo di dibattito circa la recente situazione politico-culturale della penisola.Fu il plenipotenziario Heinrich von Bellegarde che nel 1815 propose la creazione di questo spazio di incontro, aperto e libero, dove gli intellettuali italiani avrebbero potuto confrontare i diversi punti di vista sulla questione nazionale, dopo il contraccolpo vissuto durante il periodo napoleonico. Nacque pertanto la Biblioteca italiana, la quale in verità superò ben presto il progetto di giornale enciclopedico per acquisire effettiva importanza politica, confermando la stretta connessione tra la vita intellettuale e la questione nazionale nell'Italia del XIX secolo.L'obiettivo di Bellegarde, in effetti, non era politicamente neutro, la sua proposta includeva la diffusionedella simpatia per l'Austria nelle classi colteitaliane, condizionenecessaria per ottenere il sostegno finanziario del governo ufficiale per la stampa e la circolazione del giornale. Benché ci siano fonti che confermano le tendenze liberali di Bellegarde (BIZZOCCHI, 1979, p.10-11), il suo lavoro era sotto la costantesupervisione di Franz von Saurau ${ }^{6}$, il quale a sua volta giudicava le proposte del plenipotenziario austriaco "decisamente troppo per il governo di Vienna" (BIZZOCCHI, 1979, p. 11, corsivo nostro), mentre erano, in realtà, poco favorevoli agli italiani desiderosi di indipendenza.

La forza del progetto avrebbe dovuto essere guidata da personaggi di rilievo e, in effetti, gli inviti a Ugo Foscolo e a Vincenzo Monti sigillarono il prestigio agognato per la nuova pubblicazione. Con il rifiuto di Foscolo, non solo della direzione del giornale, ma soprattutto del giuramento di fedeltà all'Austria (che avrebbe dovuto fare prima di assumere l'incarico), rifiuto che costò al poeta l'esilio in Svizzera,Bellegarderiscosse una risposta affermativa da parte di Monti, in veste di consulente(non di direttore del giornale).Più tardi, la eccessiva subordinazione e il controllo da parte del governo

\footnotetext{
${ }^{6} \mathrm{Nel} 1815$ Sarau era governatore di Milano e rappresentava una delle figure austriache più conservatrici del periodo preunificazione.
} 
sull'organizzazione della pubblicazione determinò il discreto allontanamento di Vincenzo Monti, con una delega delle sue funzioni di consulente, nei primi mesi del 1816, a Giuseppe Acerbi, noto geografo e viaggiatore lombardo.Acerbi, da un lato, promosse con successo una campagna di adesioni in tutta l'Italia, dall'altro,nonostante non godesse della stessa reputazione di Foscolo, Giordani o Monti, ricevette dal governo austriaco la fiducia e il sostegno necessario. In risposta ad alcune critiche interne in merito alla sua posizione filogovernativa e alle insubordinazioni del geologo romano Scipione Breislak, collaboratore regolare del giornale, Acerbi decise di assumere la legittima amministrazione del giornale in luglio 1816, incarico detenuto in precedenza dall'editore e libraio Antonio Fortunato Stella. Nuovo proprietario della Biblioteca, Acerbi decise di trasformare la gestione del giornale -la quale poggiava sul sostegno degli abbonati-da privata a pubblica: onorando lafiducia concessa dal potere austriaco ${ }^{7}$, fece del periodico una pubblicazione a completo controllo dello Stato ${ }^{8}$. Questa strategiageneròla rottura concompilatori di maggiore importanza come Pietro Giordani e larinuncia di alcuni abbonati, ma ciò non fece desistere lo stesso Acerbi, il quale affermò: "Il nostro giornale ha mezzi, sussidii, auspicii di cui non gode alcun altro giornale" (BIZZOCCHI, 1979, p.17)9.

Scontento delle decisioni di Acerbi, Monti iniziò a idearee già nei primi mesi del 1817poteva contare su dei nomi di rilievo in suo sostegno, come quello dello storico Carlo Rosmini, del filologo Andrea Mustoxidi e delloscrittore Mario Pieri. Acerbi, a sua volta,ostacolò formalmente l'iniziativa di Monti: da un lato riuscì a impedire per un certo tempo cheil nuovo giornale

\footnotetext{
7 "L'Austria protegge la Biblioteca Italiana, e vigila il Conciliatore con una censura rigorosa" (MIGLIORINI, 2010, p.529).

${ }^{8}$ BIZZOCCHI, 1979, p.13-16 passim.

${ }^{9}$ Giuseppe Acerbi si riferiva alla situazione decadente di molti giornali e riviste letterarie di quel periodo: "Non so più nuove del Giornale di Scienze di Firenze dopo il X fascicolo e delle Novelle letterarie dopo le poche puntate uscite alla luce. Il Giornale Enciclopedico di Napoli è morto dopo l'VIII numero, quello di Parma parimenti. A Padova Brera non è comparso da molto tempo. Quello della Letteratura Italiana è il più costante [...] Lo Spettatore di qui è perdente. Quello di Brugnatelli egualmente, quello di Omodei di Medicina cava appena le spese. Vedremo cosa faranno le Effemeridi di Roma e gli Oppuscolidi Bologna" (BIZZOCCHI, ivi).
} 
ottenesse il permesso per la pubblicazione, dall'altro ebbe modo di sottoporre il periodico a profondi cambiamenti, con una notevole ridefinizione rispetto al suo progetto iniziale.

Un'altra pubblicazione che ebbe notevole presenza e partecipò attivamente alla vita intellettuale italiana del XIX secolo fuIl Conciliatore, noto anche comeFoglio Azzurro. Con la caduta di Napoleone e del Regno d'Italia, molti italiani si sentironoillusi rispetto alle promesse di autonomia, considerati il controllo dei costumi e della libertà di stampa annunciate dal governo austriaco. Sebbene alcune lotte avesseroconseguito l'annessione o l'indipendenza di alcuni stati in quel periodo, la sottomissione del Nord-Italia al dominio austriaco e il restauro di quasi tutte le antiche divisioni statali confermarono il ritorno allo stato di soggezione al potere straniero: "Gl'italiani [...] furono considerati schiavi e come schiavi aggregati ad una mal composta famiglia di popoli, sottoposti ad un regime da caserma, e gradatamente sentirono serrarsi d'intorno le catene che li legavano allo straniero" (CLERICI, 1903, p. 4). Ciononostante, unfortissimo anelito alla libertà e un'ansia di rinnovamento avevano lasciato radici profonde in Italia sin dalle invasioni napoleoniche, facendo prosperare all'interno della società un processo di ricerca di conquiste civili, di rivendicazionedei diritti del popolo e della dignità nazionale (CLERICI, 1903, p. 4). Fu proprio nella città di Milano, che comeabbiamo visto ebbe una viva partecipazione alla vita intellettuale del paese, che presero a diffondersi nuove idee, echi a loro volta dellerecenti trasformazioniavvenute nel resto dell'Europa. $\grave{E}$ in questo contesto che nel 1818 Silvio Pellico, lamentando il malessere avvenuto all'interno della Biblioteca italianapochi anni prima, suggeriva a Luigi Porro Lambertenghi di fondare una società, Conciliatore, la quale si sarebbe data un nuovo giornale, aperto alla letteratura europea, e non solo italiana (CLERICI, 1903, p.9-10). Nel giugno del 1818, uscìdunque la prima edizione deIl Conciliatore: la nuova pubblicazione cercava di suscitare e promuovere, così nei 
liberali come nei patrioti, uno spirito di discussione, proponendosi come un ambiente amichevole di dibattito sul futuro dell'Italia: "Il Conciliatore fu come il risultato delle aspirazioni più o meno consapevoli di una famiglia di poeti, di critici, di pensatori, che dopo molti tentativi trovarono l'espressione naturale delle loro idee in quel periodico [...]" (CLERICI, 1903, p.12).

Il nuovo giornale, tra $\mathrm{i}$ cui soci figuravano i conti Porro-Lambertenghi e Federico Confalonieri, Vincenzo Monti, Ludovico di Breme, Pietro Borsieri e Giovanni Berchet, rappresentò una deviazione dalla posizione decisamente moderata del romanticismo italiano, in quantodichiarava apertamente le sue tendenze estetiche innovative. In esse, allo stesso tempo, si trovavano diverse tracce del pensiero razionalista del XVIII secolo; tra i suoi letterati, alcuni provenivano dal periodico Il Caffe e dall'Accademia dei Pugni, espressioni dell'illuminismo milanese ${ }^{10}$. La censura austriaca, sempre più severa $e$ intollerante, prendeva la parola "romantico" per l'espressione "liberale", e sulla base di questa sinonimia accolsedenunce che affermavano esserci a Milano una "setta" con lo scopo di insegnare agli italiani che non erano sottomessi a nessun principio morale o religioso (CLERICI, 1903, p.205) e cheinoltre forti erano i legami tra il giornale e il movimento della Carboneria ${ }^{11}$. In quel periodo di frammentazione politica, qualsiasi tipo di minaccia alla sovranità eraimmediatamentecombattuta e gli ideali romantici venivano considerati una forma di affronto alle autorità e di cospirazione contro il dominio straniero. Iniziò dunque una serie di arresti, sotto l'accusa di associazione e di partecipazione alle insurrezioni promosse daiFederati ${ }^{12}$. Dopo un lungo

\footnotetext{
${ }_{10}$ Il periodico Il Caffe, pubblicato tra gli anni 1764 e 1766, era legato a espressioni culturali e letterarie erudite, seguendo norme e interessi di diverse Accademie e proponendosi come una voce dei conflitti politici e dell'utilità della letteratura, basata in bisogni reali (RAIMONDI, 1997, p.43).

${ }^{11} \mathrm{La}$ Carboneria fu una società segreta rivoluzionaria nata nell'allora Regno di Napoli, inizialmente in opposizione al governo napoleonico e ai suoi rappresentanti locali, Joachim Murat e Ferdinand I de Bourbon, e ulteriormente si oppose anche alle riforme austriache. Propugnava, nel periodo di dominio straniero, valori liberalie patriottici; ottenne il sostegno di intellettuali di tutto il territorio italiano, come quello del conte milanese Federico Confalonieri, uno dei fondatori de Il Conciliatore. Cfr. DITO, 1966 e PELLICO, 2012, p.25.

${ }^{12} \mathrm{Federati}$ era un'altra società segreta che organizzava e partecipava a gran parte delle manifestazioni intellettuali e insurrezionali in Piemonte nel 1821.
} 
processo concluso nel 1821, Pellico, Confalonieri, Borsieri e Piero Maroncelli ${ }^{13}$ furono arrestati, mentre Gian Domenico Romagnosi fu assolto dalle accuse. L'arrestomise fine alle attività deIl Conciliatore, che aveva già sofferto sin dall'inizio di diverse forme di limitazione.

L'esperienza de Il Conciliatore creò un intenso e riccobdialogo coninnumerevoli intellettuali e letterati italiani e stranieri i quali, dopo aver assistito alla sua tragica fine, si sentirono chiamati a continuare lesue attività. Molti progetti in questa direzione sorsero a Firenze, quasi tutti però destinati a fallire. Fino al 1816, per esempio, il Giornale di letteratura e di belle arti di Francesco Benedettisi proponeva di fare della letteratura uno strumento di formazione civile e politica degli italiani; tuttavia un suo articolo del settembre dello stesso anno, "La romanticomania, Dialogo fra Madonna, Messer lo Giornalistae Il Cavaliere", che aveva lo scopo di porre fine alla polemica iniziata con il testo di Madame de Staël nella Biblioteca Italiana ${ }^{14} \mathrm{e}$ di difendere l'estetica romantica, generò la critica più intransigente della scrittrice francese. Dopo questo episodio, l'esistenza della rivista ebbe i giorni contati.

Iniziò contemporaneamente nel 1821 un tentativo di rispondere alla dominazione austriaca, in continuità con IlConciliatore: il giornale Antologia prese forma solo nel 1822, a cura di Gian Pietro Vieusseux e Gino Capponi. Inizialmente concepita come una "scelta d'opuscoli d'ogni letteratura tradotti in italiano"15 (sul modello francese della Revue encyclopédique di antologia di letture), questo nuovo giornale prendeva ancora le distanze dal suo progetto iniziale ideato nel 1820 da Vieusseux di una "Gazzetta letteraria di Firenze"

${ }^{13}$ Il caso dell'arresto di Pellico, Borsieri, Maroncelli e Confalonieri ha suscitato la reazione di molti letterati. Inizialmente condannati a morte dallimperatore austriaco, le loro sentenze sono state commutate in anni di carcere duro. Borsieri e Confalonieri, dopo un lungo periodo di carcere, sono esiliati negli Stati Uniti e Maroncelli, a causa di una grave malattia e dell'amputazione di una gamba, è andato in esilio in Francia e, anni dopo, si è trasferito negli Stati Uniti (CLERICI, 1903, p.211-216 passim).

${ }^{14} \mathrm{Ci}$ si riferisce a Sulla maniera e l'utilità delle traduzioni, tradotto da Pietro Giordani e pubblicato il $1^{\circ}$ gennaio 1816 nel numero 6 della rivista Biblioteca Italiana. Il testostaelianoha avuto un ruolo fondamentale nella scena letteraria italiana, in quanto è stato lo stimolo perché gli intellettuali prendessero posizione in merito alle questioni estetiche nel più ampio contesto della diffusione del romanticismo in Italia.

${ }^{15}$ DESIDERI, in TORTORELLI, 2002, p.127-128. 
(SAVINI, 1974, p.11), come sede di dibattito su letteratura, belle arti e teatro, oltre a temi di economia, morale e leggi. La rivista acquisì nel tempo un profilo più ampio, con un approccio che includeva le scienze e un taglio pragmatico, non solo ed esclusivamente teorico.

Le collaborazioni straniere sono state respinte nella rivista, presentando nel suo primo anno solo contributi di autori italiani, rafforzatidalla revisione di redattori toscani e da un intenso sforzo critico edidatticodi tutto il gruppo di intellettuali coinvolti (SAVINI 1974 p.11-12). Ciò significa che l'impegno educativo alla società civile era uno dei pilastri della Antologia, che discorreva anche sulle questioni politiche che animavano l'intero contesto artistico italiano. Neicontributi di personalità come Pietro Giordani, Giacomo Leopardi, Niccolò Tommaseo e Giuseppe Montani non mancarono, a differenza de Il Conciliatore, critichealla estetica romanticae alle trasformazioni stilistiche che essa proponeva, fattoche non permette tuttavia di affermare che i contenutifossero completamente privi di elementi romantici oppure chetrattassero esclusivamente di temi classicisti.

Nonostante l'esigenza intellettuale dell'Antologia fosse quella di valorizzare la tradizione, i letterati che collaborarono con la rivista fiorentina dichiararono una necessità sempre più viva di dialogare con le letterature straniere. Prova di tale bisogno sono le citazioni di autori stranieri in alcuni saggi: Goethe, Byron, Stendhal, Scott e Chateaubriand (SAVINI 1974 p.15). La rivista venne sospesa nel 1833, dopo una denuncia presentata dalla gazzetta di Modena La voce della verità, rappresentante della Controrivoluzione ${ }^{16}$ e, per certi aspetti, simpatizzante del potere austriaco. Niccolò Tommaseo, in risposta alle accuse, scrisse nel 1835 una lunga protesta, di cui riportiamo un estratto:" $\mathrm{E}$ poiché G. P. Vieusseux, con l'assenso del governo Toscano chiamava bugiarda e calunniatrice La voce della verità, già colpevole d'altre calunnie, e

16 La Controrivoluzione fu un movimento intellettuale e militare che combatteva i principi democratici di Napoleone e difendeva la restaurazione dell'ordine esistente prima dell'invasione francese. Uno dei suoi più noti sostenitori fu Monaldo Leopardi, padre del poeta Giacomo Leopardi. 
d'altre bugie [...] doversi l'Antologia giudicare secondo i giudizii di chi non ne fu mai parte, e mal la conobbe" (GALVANI, 1931, p.8).

Concludendo, abbiamo percorso brevemente la storia di queste pubblicazioni nell'intento di dimostrare come il progetto ideologico a cui ognunasi trovava legata abbia contribuito a determinare il ruolo che ciascuna di esse ha avuto in seno alla produzione critica italiana e alla descrizione del campo di applicazione di elementi costitutivi della cultura, principalmente la letteratura. Non dimentichiamo, inoltre, che grazie all'attività di queste riviste gran parte delle teorizzazioni e dei dibattiti sulla natura e sullo sviluppo della cultura letteraria italiana si manifestòin un riccodialogo di incontri e confronti interni ed esterni. Il conflitto tra tradizione e innovazione fu straordinariamente produttivo per entrambe le parti, in quanto i progetti estetici furono rivisitati e rivalutati alla luce di nuove idee derivanti da altri paesi dell'Europa oppure delineate all'interno della intellighenzia italiana.

\section{Riferimenti bibliografici}

BIZZOCCHI, Roberto. La "Biblioteca Italiana" e la cultura della Restaurazione (1816-1825). Milano: Franco Angeli, 1979.

CLERICI, Edmond. Il "Conciliatore": periodico milanese (1818-1819). Pisa: Tipografia Successori FF. Nistri, 1903.

DESIDERI, Laura. La biblioteca del Gabinetto Vieusseux negli anni dell' 'Antologia'. In: TORTORELLI, Gianfranco. Biblioteche nobiliari e circolazione del libro tra Settecento e Ottocento. Atti del Convegno nazionale di studio, Perugia, Palazzo Sorbello, 29-30 giugno 2001. Bologna: Pendragon, 2002.

DITO, Oreste. Massoneria, carboneria ed altre società segrete nella storia delRisorgimento italiano. Bologna: Arnaldo Forni, 1966.

FUBINI, Mario. Romanticismo italiano. Saggi di storia della critica e della letteratura italiana. Bari: Giuseppe Laterza \& Figli, 1953. 
GALVANI, Cesare Carlo. La gazzetta La voce della verità condannata a morte ignominiosa senza appello con sentenza proferita a Parigi nell'aprile $1835 \mathrm{da}$ Ser Cotale Niccoló Tommaseo e compagni per strage commessa dell'Antologia e per attenti contro la liberalesca settaria sovrana canaglia. Filadelfia: Harvard Press, 1931.

MIGLIORINI, Bruno. Storia della lingua italiana. Milano: Bompiani, 2010.

PELLICO, Silvio. Lettere al conte milanese Federico Confalonieri (18311840).Organização de Cristina Contilli. Raleigh: Lulu, 2012.

RAIMONDI, Ezio. Romanticismo italiano e romanticismo europeo. Milano: Bruno Mondadori, 1997.

SAVINI, Marta. Riviste ottocentesche e la storia della critica. Roma: Bulzoni, 1974.

VIGORELLI, Giancarlo (Org.). Edirione nazionale ed europea delle opere di Alessandro Manzoni. Testi criticamente riveduti e commentati. Volume 13. Arbizzano: Tipografia Valdonega, 2008. 


\title{
A atuação das revistas literárias italianas na primeira metade do Ottocento ${ }^{17}$
}

\author{
Gisele Batista da Silva ${ }^{18}$ \\ Universidade Federal do Rio de Janeiro \\ gisabats@gmail.com
}

RESUMO: Neste artigo, analisamos a criação, atuação e o papel de algumas revistas literárias italianas criadas na primeira metade do século XIX, representadas principalmente pela Biblioteca Italiana, Il Conciliatore e Antologia. Destacamos no estudo proposto as relações político-culturais estabelecidas entre seus fundadores e interlocutores e evidenciamos a participação e o aporte desses periódicos na difusão ou crítica à questão romântica, que ganhou destaque e importância na Itália durante o período em questão. Examinamos, ainda, algumas transformações que essas revistas exerceram nos círculos de literatos italianos, desempenhando papel fundamental na tomada de posição desses intelectuais, principalmente no que diz respeito às questões culturais da Itália oitocentista.

Palavras-chave: Romantismo italiano. Revistas literárias. Biblioteca Italiana. Il Conciliatore. Antologia.

ABSTRACT: The purpose of this article is to analyse the creation, performance and role of some Italian literary magazines created in the first half of the 19th century, mostly represented by the Biblioteca Italiana, Il Conciliatore and Antologia. It emphasizes the political-cultural relations established between its founders and interlocutors and shows the participation and the contribution of these periodicals in the diffusion of the romantic issue that gained prominence and importance in Italy in the analysed period. It have also examines some of the changes on Italian writer's circles influenced by these magazines, which revealed that they have played a fundamental role in shaping intellectual positions regarding the cultural issues of nineteenthcentury Italy.

Keywords: Italian Romanticism. Literary magazines. Biblioteca Italiana. Il Conciliatore. Antologia.

\footnotetext{
17 Tradução do próprio autor.

${ }^{18}$ Doutora em Literatura italiana pela UFRJ, Mestre em Literatura brasileira (UERJ) e em Didattica dell'italiano a stranieri (Ca' Foscari). Atualmente, é Professora Adjunta de Língua italiana na UFRJ e participa do GT da Anpoll "Estudos da tradução".
} 
Tentando una riforma era nostro debito di indicare con precisionerigorosa le vere fonti e i soggetti della nuova letteratura [...] Noi volevamo congiungere in alleanza la letteratura d'Italia collo spirito del Secolo.

Alessandro Manzoni Notizia sul Romanticismo in Italia

Em carta escrita a Charles-Claude Fauriel em 1820, Alessandro Manzoni compartilhou com o amigo francês sua revisão sobre os efeitos e as ressonâncias do movimento romântico na Itália. Para o literato milanês, o Romantismo italiano não havia representado a introdução de "un intero sistema di teorie Germaniche", mas foi construído "pezzo per pezzo e senz'aver un precedente disegno" (VIGORELLI, 2008, p.201), inicialmente nas páginas dos periódicos Biblioteca Italiana e Conciliatore. A reflexão de Manzoni sobre os objetivos do movimento romântico na Itália evidencia a sua peculiar situação em relação ao resto da Europa, uma vez que o seu romantismo ainda guardava em um "falso Italianismo" (VIGORELLI, 2008, p.203) a justificativa para esconder um estado de ignorância e inércia, que geravam imobilidade e atraso cultural. Dos equívocos listados pelo escritor e dramaturgo lombardo nessa mesma carta, destacamos aquele em que se presumia que o povo italiano, mais do que qualquer outro, fosse dotado de uma notável imaginação, característica que lhe outorgava uma posição incomparavelmente superior ao resto da Europa. Esse tipo de pensamento, representado por "frases parasitas" propaladas em meios acadêmicos (VIGORELLI, 2008, p.204), refletia uma conjuntura histórico-social bastante complexa (e também dela era reflexo) ${ }^{19}$, na qual estava implicada uma conflituosa convivência entre tradição e inovação. Em resposta a esse contexto, o projeto manzoniano desejava examinar as fontes, afastando-se das sentenças enganosas ${ }^{20}$, e agregar à literatura italiana o cenário europeu em

19“[...] la Letteratura, essendo inerente allo Stato sociale di un popolo, ne segue necessariamente le fasi, ne raccoglie lo spirito, e ne diviene la più bella espressione" (VIGORELLI, 2008, p.204).

${ }^{20}$ Entre elas: "non solo i quattro grandi nostri Classici, ma tutta l'infinita serie sinonima de' trecentisti e de' cinquecentisti, toccarono l'apice della perfezione "; "quanto più una produzione 
transformação, que reavaliava a tradição e propunha novos caminhos e objetivos para a literatura nacional.

Outros escritos também revelaram a consciência dessas mudanças, que direcionavam a Itália à discussão de renovados conteúdos e formas culturais: a Lettera semiseria di Grisostomo al suo figlinolo, de Berchet; o Sermone sulla mitologia, de Monti; a Lettre à M. Chauvet sur l'unité de temps et de lieu dans La tragédie, de Manzoni ou, ainda, os diversos textos do "filósofo do Romantismo italiano", Ermes Visconti ${ }^{21}$. Essas manifestações críticas deram voz a aspirações de cunho literário, mas também difundiram os projetos ideológicos desses intelectuais, visto que todos estavam diretamente envolvidos na tarefa de dar atributo, consistência e visibilidade a uma lacuna na história (literária) italiana: uma identidade.

A "Italia italiana e non latina"22 era cobiçada pelos românticos em oposição ao nivelamento cultural entre gregos, latinos e italianos, e representou uma espécie de mote divisor de águas na polêmica de antigos e modernos, mas sobretudo entre a parte da Itália de orientação moderna e a outra, de tendência classicista, com todas as implicações ideológicas que esse tema pudesse sugerir. $\mathrm{E}$ a arena de debate, onde os argumentos de ambas as apreciações foram expressos, estava bem representada em revistas e jornais literários, principalmente nas publicações da Biblioteca Italiana, de Il Conciliatore e da Antologia.

Milão, capital do Regno d'Italia entre 1805 e 1814, centro respeitável da vida intelectual italiana, assimilou durante esse período o que de melhor se produziu em matéria intelectual e cultural. Era urgente, portanto, a criação de um periódico que filtrasse e difundisse essas produções e que se colocasse,

nuova somiglia un'antica [...] tanto è migliore"; "noi discendendo da' Romani siamo il Popolo primogenito, il popolo nobile dell'Europa" (VIGORELLI, 2008, p.203).

${ }^{21}$ A denominação dada pelo amigo Goethe manifestava sua aposta no talento crítico de Visconti, esperando que colocasse fim à polêmica romântica na Itália e aos conflitos dela decorrentes. Cf. FUBINI, 1953, p.10.

22 Di Breme, em carta de maio de 1816 a Diodata Saluzzo, na qual pede autorização para publicar sua defesa a Madame de Staël (FUBINI,1953, p.16). 
além disso, como território de debate sobre a recente situação político-cultural da península. Assim, o plenipotenciário Heinrich von Bellegarde se propôs a criar em 1815 um espaço de encontro para que intelectuais italianos, de forma aberta e livre, pudessem abordar os diferentes pontos de vista sobre a questão nacional, após o abalo vivido durante o período napoleônico. $\mathrm{O}$ resultado dessa exigência foi que a Biblioteca Italiana transcendeu o projeto de um jornal enciclopédico para adquirir efetiva importância política, confirmando a estreita conexão entre vida intelectual e a questão nacional italiana no século XIX. A grandeza do projeto deveria ser dirigida por personagens de igual vulto e, de fato, os convites a Ugo Foscolo e Vincenzo Monti selaram o absoluto prestígio desejado para a nova publicação italiana. O objetivo de Bellegarde, é mister destacar, não era politicamente neutro, e sua proposta incluía difundir a simpatia pela Áustria nas classes cultas italianas, condição imperiosa para que obtivesse o apoio financeiro oficial do governo para a impressão e a circulação do jornal. Embora haja fontes que confirmem as tendências liberais de Bellegarde (BIZZOCCHI, 1979, p.10-11), seu trabalho era constantemente supervisionado por Franz von $\mathrm{Saurau}^{23}$, para quem as propostas do plenipotenciário austríaco "era [no] decisamente troppo per il governo di Vienna" (BIZZOCCHI, 1979, p.11, grifo nosso), e contrariamente pouco favoráveis aos italianos desejosos de independência. Com a recusa de Foscolo, não apenas da direção do jornal, mas sobretudo do juramento de fidelidade à Áustria que deveria fazer antes de assumir o cargo (logo depois da negativa, Foscolo se exilou na Suíça), Bellegarde teve posteriormente resposta afirmativa de Monti, que iniciou suas atividades de assessor - não de diretor do jornal. Mais tarde, a excessiva subordinação e controle do governo sobre a organização cultural da publicação fez com que Vincenzo Monti se afastasse discretamente das responsabilidades assumidas, delegando no início de 1816 a condução de suas atividades de assessoria a Giuseppe Acerbi, conhecido

\footnotetext{
${ }^{23}$ Em 1815, Sarau era governador de Milão e representava uma das figuras austríacas mais conservadoras do período pré-unificação.
} 
geógrafo e viajante lombardo. Acerbi iniciou, então, o envio, para toda a Itália, de convites de associação e colaboração ao jornal, de onde recebeu poucas recusas e inúmeras adesões. $\mathrm{O}$ novo assistente do jornal, mesmo que não desfrutasse da mesma reputação de um Foscolo, Giordani ou Monti, ganhou do governo austríaco a confiança e a certeza de fidelidade e idoneidade na condução das atividades da publicação. Pressionado internamente por seu apoio ao governo e por algumas insubordinações do geólogo romano Scipione Breislak, regular colaborador do jornal, Acerbi decidiu assumir a legítima administração do jornal em julho de 1816, que antes pertencia ao editor e livreiro Antonio Fortunato Stella, tornando-se o novo proprietário da Biblioteca e reconduzindo a gestão privada do jornal - que antes mantinha grande parte de seu sustento com o dinheiro dos associados - para a completa proteção, comando e controle do $\mathrm{Estado}^{24}$, fazendo valer a credibilidade que lhe fora depositada pelo poder austríaco ${ }^{25}$. Essa manobra gerou o rompimento com compiladores do vulto de Pietro Giordani e a renúncia de alguns associados, mas Acerbi mantinha-se otimista, certo de que "Il nostro giornale ha mezzi, sussidii, auspicii di cui non gode alcun altro giornale" ${ }^{26}$. Descontente com as últimas decisões de Acerbi, Monti começou a idealizar com alguns aliados um novo jornal que se opusesse à Biblioteca Italiana e sorrateiramente conseguiu angariar simpatizantes para a sua causa nos primeiros meses de 1817: nomes como o do historiador Carlo Rosmini, do filólogo Andrea Mustoxidi e do literato Mario Pieri. Giuseppe Acerbi reagiu à empreitada de Monti, obstaculizando formalmente a sua iniciativa e, com efeito, conseguiu impedir que o novo jornal obtivesse permissão de publicação. Toda a

24BIZZOCCHI, 1979, p.13-16 passim.

25"L'Austria protegge la Biblioteca Italiana, e vigila il Conciliatore con una censura rigorosa" (MIGLIORINI, 2010, p.529).

${ }^{26 B I Z Z O C C H I, ~ 1979, ~ p .17 . ~ G i u s e p p e ~ A c e r b i ~ r e f e r i a-s e ~ a ̀ ~ s i t u a c ̧ a ̃ o ~ d e c a d e n t e ~ d e ~ m u i t o s ~ j o r n a i s ~ e ~}$ revistas literárias naquele período: "Non so più nuove del Giornale di Scienze di Firenze dopo il X fascicolo e delle Novelle letterarie dopo le poche puntate uscite alla luce. Il Giomale Enciclopedico di Napoli è morto dopo l'VIII numero, quello di Parma parimenti. A Padova Brera non è comparso da molto tempo. Quello della Letteratura Italiana è il più costante [...] Lo Spettatore di qui è perdente. Quello di Brugnatelli egualmente, quello di Omodei di Medicina cava appena le spese. Vedremo cosa faranno le Effemeridi di Roma e gli Oppuscoli di Bologna" (BIZZOCCHI, ivi). 
estratégia de Acerbi condicionou o periódico a sofrer profundas mudanças, que o desviaram decisivamente do projeto inicial.

Outra publicação que teve notável presença e que participou ativamente da vida intelectual da Itália oitocentista foi Il Conciliatore, também conhecido por Foglio Azzurro. Com a queda de Napoleão e do Regno d'Italia, muitos italianos sentiram-se iludidos com uma promessa de autonomia, respeito aos costumes e à liberdade de imprensa anunciados pelo governo austríaco. Embora algumas lutas tenham conseguido a anexação ou a independência de alguns estados nesse período, a clara submissão do norte da Itália ao domínio austríaco e o restabelecimento de quase todas as antigas divisões estatais confirmaram o retorno ao estado de sujeição ao poder estrangeiro: "Gl'italiani [...] furono considerati schiavi e come schiavi aggregati ad una mal composta famiglia di popoli, sottoposti ad un regime da caserma, e gradatamente sentirono serrarsi d'intorno le catene che li legavano allo straniero" (CLERICI, 1903, p.4). Apesar disso, um espírito de liberdade e, principalmente, de renovação haviam deixado raízes na Itália desde as investidas napoleônicas, fazendo prosperar no interior da sociedade um processo de busca de conquistas civilizatórias, de reivindicação dos direitos do povo e de dignidade nacional (CLERICI, 1903, p.4). E da cidade de Milão, que como vimos teve uma viva participação na vida intelectual do país, despertaram novas ideias, ecoadas das últimas transformações acontecidas no resto da Europa. Assim, em 1818, Silvio Pellico falava da iniciativa de Luigi Porro-Lambertenghi em fundar uma sociedade, a Conciliatore, e, lamentando o mal-estar ocorrido no interior da Biblioteca Italiana poucos anos antes, apresentava-a como incentivo à participação do projeto de um novo jornal, cujo objetivo era a análise de livros europeus, e não apenas italianos (CLERICI, 1903, p.9-10). Finalmente, em junho de 1818, saiu o primeiro fascículo intitulado Il Conciliatore, tendo como sócios do jornal os condes Porro-Lambertenghi e Federico Confalonieri, além de Monti, Di Breme, 
Pietro Borsieri e Giovanni Berchet. A nova publicação procurou suscitar e promover, tanto nos liberais como nos patriotas, um espírito de discussão, garantindo no periódico um ambiente amigável e sociável de debate sobre o futuro da Itália: "Il Conciliatore fu come il risultato delle aspirazioni più o meno consapevoli di una famiglia di poeti di critici di pensatori, che dopo molti tentativi trovarono l'espressione naturale delle loro idee in quel periodico [...]" (CLERICI, 1903, p.12).

O novo jornal representou um desvio da postura decididamente moderada do romantismo italiano, porquanto declarasse abertamente suas tendências estéticas inovadoras, ao mesmo tempo em que ainda se encontravam vários rastros do pensamento racionalista do século XVIII entre seus literatos, alguns herdados do periódico Il Caffêe da Accademia dei Pugni, dos quais participaram intelectuais iluministas milaneses ${ }^{27}$. A atuante censura austríaca, cada vez mais rigorosa e intolerante, reconhecia na palavra "romântico" a expressão "liberal" e , baseada nessa sinonímia, acatou as denúncias de que em Milão havia uma "seita" com o escopo de ensinar aos italianos que eles não eram submissos a nenhum princípio moral ou religioso (CLERICI, 1903, p.205) e, ainda, de que esse grupo mantinha ligação com o movimento de Carboneria ${ }^{28}$. Naquele período de fragmentação política, todo e qualquer tipo de ameaça à soberania era prontamente combatida pelo governo austríaco, e os ideais românticos encarnavam uma forma concreta de intimidação à autoridade e de conjura ao domínio estrangeiro. Iniciou-se, então, uma série de prisões, sob a acusação de conspiração e participação em

\footnotetext{
27 O periódico Il Caffe, publicado entre os anos de 1764 e 1766, estava ligado a expressões culturais e literárias eruditas, seguindo normas e interesses de diferentes Academias e lançando apelos a conflitos políticos e a uma literatura útil e fundamentada em necessidades reais (RAIMONDI, 1997, p.43).

28 A Carboneria foi uma sociedade secreta revolucionária nascida no então Regno di Napoli, inicialmente como oposição ao governo napoleônico e a seus representantes locais, Joachim Murat e Ferdinand I de Bourbon, e que posteriormente opôs-se também às reformas austríacas. Propagava, naquele período de domínio estrangeiro, valores liberais e patrióticos e obteve apoio de intelectuais de todo o território italiano, como do conde milanês Federico Confalonieri, um dos idealizadores de Il Conciliatore. Cf. DITO, 1966 e PELLICO, 2012, p.25.
} 
insurreições dos Federati $2^{29}$ : depois de um longo processo terminado em 1821, foram presos Pellico, Confalonieri, Borsieri e Piero Maroncelli ${ }^{30}$, enquanto que Gian Domenico Romagnosi foi absolvido das acusações. Esse ataque deu fim às atividades de Il Conciliatore, que já havia sofrido desde o seu surgimento diversas proibições.

A experiência de Il Conciliatore criou intenso e rico diálogo com inúmeros intelectuais e literatos italianos e estrangeiros, que, tendo assistido ao seu trágico fim, sentiram-se convocados a dar continuidade às suas atividades. Muitos projetos nesse sentido nasceram em Florença, quase todos extintos antes mesmo que germinassem. Até 1816, por exemplo, o Giornale di letteratura e di belle arti de Francesco Benedetti se propunha a conduzir a literatura à educação civil e política dos italianos, mas um artigo seu de setembro daquele ano, "La romanticomania, Dialogo fra Madonna, Messer lo Giornalista e Il Cavaliere", que pretendia encerrar a polêmica iniciada com o texto de Madame de Staël na Biblioteca Italiana ${ }^{31}$ e defender a estética romântica, acabou gerando as críticas mais inflexíveis à escritora francesa. Após esse episódio e suas diversas reações, a existência do periódico estava naturalmente com os dias contados.

Iniciou-se, contemporaneamente, um movimento reformador que sucedeu as atividades interrompidas do Conciliatore e que pretendia, assim como o jornal milanês, ser mais uma tentativa de resposta à dominação austríaca. A idealização da Antologia nasceu em 1821, por empenho de Gian

\footnotetext{
${ }^{29}$ Federati foi outra sociedade secreta que organizou e participou de grande parte das manifestações intelectuais e insurrecionais no Piemonte em 1821.

30 O delicado caso de Pellico, Borsieri, Maroncelli e Confalonieri suscitou a manifestação de vários de seus colegas literatos. Inicialmente condenados à morte pelo imperador austríaco, suas penas foram comutadas em anos de prisão rigorosa. Borsieri e Confalonieri, depois de longo período de cárcere, se exilaram nos Estados Unidos, e Maroncelli, em decorrência de uma grave doença e da amputação de uma perna, exilou-se na França e, anos mais tarde, mudou-se também para os Estados Unidos (CLERICI, 1903, p.211-216 passim).

31 Estamos nos referindo a Sulla maniera e l'utilità delle tradurioni, traduzido por Pietro Giordani e publicado em $1^{\circ}$ de janeiro de 1816 no número 6 da revista Biblioteca Italiana. Este texto staeliano teve papel fundamental no ambiente literário italiano, na medida em que foi o estopim para que intelectuais se posicionassem publicamente sobre suas opções estéticas diante do avanço do Romantismo na Itália.
} 
Pietro Vieusseux e Gino Capponi, mas só em 1822 foi publicado seu primeiro fascículo, contendo uma diretriz mais definida para o cenário intelectual italiano. Declarando seu objetivo de analisar assuntos dos mais variados temas, predominantemente científicos e literários, ao invés de apresentar, como havia planejado no ano anterior, uma "scelta d'opuscoli d'ogni letteratura tradotti in italiano"32 (tal como o modelo francês da Revue enciclopédique de antologia de leituras), essa nova expressão do jornal ainda se distanciava do projeto inicial idealizado por Vieusseux, em 1820, de uma Gazzetta letteraria di Firenze (SAVINI, 1974, p.11). A finalidade inaugural de Vieusseux para a Gazzetta teria sido debater sobre literatura, belas artes e teatro, além de temas sobre economia, moral e leis, assumindo em 1822, na Antologia, um campo de discussão bem mais amplo e científico. Sob essa nova ótica, mais científico-prática (e não rigorosamente teórica), as colaborações estrangeiras foram recusadas na nova revista, que apresentou no seu primeiro ano apenas contribuições de autores italianos, fortalecidas pelo acompanhamento de redatores toscanos e por um intenso esforço crítico e didático de todo o grupo de intelectuais envolvido (SAVINI, 1974, p.11-12). Isso quer dizer que o empenho educativo sobre a sociedade civil era um dos pilares da Antologia, que acabava por afluir também em temas políticos que animavam todo o contexto artístico italiano. Nas contribuições de personalidades como Pietro Giordani, Giacomo Leopardi, Niccolò Tommaseo e Giuseppe Montani não faltaram, diferentemente de Il Conciliatore, críticas à estética romântica e às transformações estilísticas que propunha, fato insuficiente, entretanto, para afirmar que fossem completamente destituídas de elementos românticos ou que versassem exclusivamente sobre temas classicistas.

Embora a exigência intelectual da Antologia fosse de valorização da tradição, os literatos que colaboraram com a revista florentina declararam uma

32 DESIDERI, in TORTORELLI, 2002, p.127-128. 
necessidade cada vez mais viva de dialogar com as literaturas estrangeiras. Prova disso são as citações de autores estrangeiros em alguns artigos, nomes como Goethe, Byron, Stendhal, Scott e Chateaubriand (SAVINI, 1974, p.15). A revista foi suspensa em 1833, depois de uma denúncia feita pela gazeta de Modena La você della verità, representante da Controrivoluzione ${ }^{33}$ e, em certos tópicos, simpatizante do poder austríaco. Niccolò Tommaseo, em reação às acusações, escreveu em 1835 um longo protesto, cujo brevíssimo trecho reproduzimos: "E poiché G. P. Vieusseux, con l'assenso del governo Toscano chiamava bugiarda e caluuniatrice la Voce della Verità, già colpevole d'altre caluunie, e d'altre bugie [...] doversi l'Antologia giudicare secondo i giudizii di chi non ne fu mai parte, e mal la conobbe" (GALVANI, 1931, p.8).

Concluindo, percorremos brevemente a história dessas publicações, por acreditar que o projeto ideológico a que cada uma estava vinculada, colaborou na determinação do trabalho e do papel que desempenharam para a produção critica italiana e para a descrição das atribuições de elementos componentes da cultura, principalmente a literatura. Não nos esqueçamos, ainda, que foi na atividade desses periódicos que grande parte das teorizações e debates sobre a natureza e o encaminhamento da cultura literária italiana foi manifestada, um rico diálogo de encontros e confrontos internos e externos. O enfrentamento entre tradição e inovação foi extraordinariamente produtivo para ambos os lados, na medida em que os projetos estéticos foram revisitados e reavaliados à luz de novas ideias, advindas de outros países da Europa ou delineadas no interior da intelectualidade italiana.

\section{Referências Bibliográficas}

BIZZOCCHI, Roberto. La "Biblioteca Italiana" e la cultura della Restaurazione (1816-1825). Milano: Franco Angeli, 1979.

33 A Controrivolurione foi um movimento intelectual e militar, que combatia os preceitos democráticos napoleônicos e disputava o restabelecimento da ordem existente antes da invasão francesa. Um de seus célebres simpatizantes foi Monaldo Leopardi, pai do poeta Giacomo Leopardi. 
CLERICI, Edmond. Il "Conciliatore": periodico milanese (1818-1819). Pisa: Tipografia Successori FF. Nistri, 1903.

DESIDERI, Laura. La biblioteca del Gabinetto Vieusseux negli anni dell' 'Antologia'. In: TORTORELLI, Gianfranco. Biblioteche nobiliari e circolazione del libro tra Settecento e Ottocento. Atti del Convegno nazionale di studio, Perugia, Palazzo Sorbello, 29-30 giugno 2001. Bologna: Pendragon, 2002.

DITO, Oreste. Massoneria, carboneria ed altre società segrete nella storia del Risorgimento italiano. Bologna: Arnaldo Forni, 1966.

FUBINI, Mario. Romanticismo italiano. Saggi di storia della critica e della letteratura italiana. Bari: Giuseppe Laterza \& Figli, 1953.

GALVANI, Cesare Carlo. La gazzeta $L a$ voce della verità condannata a morteignominiosa senza appello con sentenza proferita a Parigi nell'aprile 1835 da Ser Cotale Niccoló Tommaseo e compagni per strage commessa dell'Antologia e per attenti contro la liberalesca settaria sovrana canaglia. Filadelfia: Harvard Press, 1931.

MIGLIORINI, Bruno. Storia della lingua italiana. Milano: Bompiani, 2010.

PELLICO, Silvio. Lettere al conte milanese Federico Confalonieri (1831-1846). Organização de Cristina Contilli. Raleigh: Lulu, 2012.

RAIMONDI, Ezio. Romanticismo italiano e romanticismo europeo. Milano: Bruno Mondadori, 1997.

SAVINI, Marta. Riviste ottocentesche e la storia della critica. Roma: Bulzoni, 1974.

VIGORELLI, Giancarlo (Org.). Edirione nazionale ed europea delle opere di Alessandro Manzoni. Testi criticamente riveduti e commentati. Volume 13. Arbizzano: Tipografia Valdonega, 2008. 